\title{
Carta al director en relación al artículo "Cáncer de próstata de alto riesgo y diseminado (Documento de Consenso de Recomendaciones)"
}

\author{
A. Berenguer Sánchez, A. Páez Borda \\ Hospitales Universitario de Getafe y de Fuenlabrada. \\ Actas Urol Esp 2006; 30 (1): 96-98
}

\section{Sr. Director:}

La reciente aparición en Actas (Actas Urol Esp 2005;29(9):826-827) de un documento de consenso sobre algunos aspectos del cáncer de próstata (Cáncer de próstata de alto riesgo y diseminado. Documento de Consenso de Recomendaciones) constituye una iniciativa interesante de la Asociación Española de Urología.

Sin embargo, ese documento merece algún comentario:

- En primer lugar, la METÓDICA observada en su elaboración no aparece especificada:

1. Las motivaciones y objetivos del estudio deben de quedar claramente recogidos en el preámbulo del documento.

2. La sistemática observada en su elaboración debe de aparecer detallada; la elaboración de documentos de similares características por organizaciones científicas relevantes es siempre precedida de profundas revisiones sistemáticas de la literatura. En esas revisiones se detallan las fuentes de información, y se enumeran los criterios de selección de los artículos considerados para la revisión. Entre los capitulos relevantes de las revisiones sistemáticas se encuentra la declaración de las fuentes de financiación (imprescindibles en un estudio de esta naturaleza) y los eventuales conflictos de intereses de los participantes. En todos los casos, una revisión sistemática incluirá el listado completo de artículos y documentos objeto de revisión en el capítulo de referencias.

3. La selección de los expertos participantes en una conferencia de consenso es esencial para asegurar una deliberación equilibrada y garantizar la credibilidad del documento final. En el grupo deben de estar representados investigadores, profesionales sanitarios, y expertos en metodología (epidemiólogos y bioestadísticos) y ética. El documento Cáncer de próstata de alto riesgo y diseminado. Documento de Consenso de Recomendaciones debería de especificar los argumentos utilizados en la selección de los expertos.

- En segundo lugar, las RECOMENDACIONES relacionadas con el tratamiento de algunas formas de cáncer avanzado son inaceptables sin una discusión pormenorizada; una búsqueda en PubMed utilizando los términos "zoledronic acid AND prostate cancer" proporciona 125 artículos; si bien algunas revisiones adecuadamente estructuradas demuestran un efecto beneficioso derivado del uso del ácido zoledrónico en algunas formas de cáncer de próstata avanzado ${ }^{1-3}$, esa conclusión no es universalmente compartida ${ }^{4-8}$.

- Finalmente, el lector crítico es conocedor de que la CALIDAD DE LA INFORMACIÓN CIENTÍFICA se jerarquiza en base a los sesgos (en otras palabras, desviaciones o errores) que lleva implícita; así, las revisiones sistemáticas de ensayos clínicos randomizados ocupan el lugar más elevado en esa jerarquía al acarrear el menor riesgo de sesgos. Los estudios no randomizados, las series de casos, los estudios observacionales y, por último, la opinión de los expertos, ocupan, en ese orden, los siguientes puestos en la estructura jerárquica de la evidencia científica ${ }^{9}$.

En definitiva, bienvenidas iniciativas como la presente. No obstante, en la era de la información y de la metodología científica el producto resulta manifiestamente mejorable. 


\section{REFERENCIAS}

1. Saad F, Gleason DM, Murray R, Tchekmedyian S, et al. A randomized, placebo-controlled trial of Zoledronic acid in patients with hormone- refractory metastatic prostate carcinoma. J Natl Cancer Inst 2002;94:1458-1468.

2. Saad F, Schulman C. Role of Bisphosphonates in Prostate Cancer. European Urology 2004;45:26-34.

3. Saad F, Karakiewicz P, Perrotte P. The role of bisphosphonates in hormone-refractory prostate cancer. World $\mathrm{J}$ Urol 2005;23:14-18.

4. Canil CM, Tannock IF. Should Bisphosphonates Be Used Routinely in Patients With Prostate Cancer Metastatic to Bone? J Natl Cancer Inst 2002;94(19): 1422-1423.

5. Atkins C, Rosenthal M, Berruti A, Tucci M, et al. A randomized, placebo-controlled trial of zoledronic acid in patients with hormone-refractory metastatic prostate carcinoma. J Natl Cancer Inst 2003;95(4):332.

6. Tu SM, Lin SH, Logothetis C. A randomized, placebo-controlled trial of zoledronic acid in patients with hormonerefractory metastatic prostate carcinoma. J Natl Cancer Inst 2003;95(15):1174-1175.

7. Winquist E, Berry S. Re: A Randomized, placebo-controlled trial of zoledronic acid in patients with hormone-refractory metastatic prostate carcinoma.J Natl Cancer Inst. 2004 Aug 4;96(15): 1183 .

8. Tanvetyanon T. Long-term efficacy of zoledronic acid for the prevention of skeletal complications in patients with metastatic hormone-refractory prostate cancer. J Natl Cancer Inst 2005;97(1):70-71.

9. Harris RP, Helfand M, Woolf $\mathrm{SH}$, Lohr $\mathrm{KN}$ et al for the Methods Work Group, Third U.S. Preventive Services Task Force. Current methods of the U.S. Preventive Services Task Force: a review of the process. Am $J$ Prev Med 2001;20(3S):21-35.

Fdo. Dres. A. Berenguer y A. Páez

\section{RESPUESTA A LA CARTA DE LOS DRES. A. BERENGUER Y A. PÁEZ}

En primer lugar agradezco que sea apreciada la iniciativa nacida en el seno del Grupo de Urología Oncológica ya que uno de los varios objetivos propuestos al asumir la responsabilidad del mismo, era crear documentos de opinión de amplio consenso en distintos aspectos conflictivos de la patología tumoral urológica, y éste es el primero de una serie que se irán publicando.

Sin duda son lógicas las observaciones de los colegas ya que en el documento no hemos hecho, explícitamente, mención de los aspectos metodológicos con los que se elaboró el documento, y que certeramente reseñan.

Mi trayectoria profesional y académica se ha realizado siempre bajo una muy cuidada y precisa metodología de estudio y trabajo que exijo cumplan todos los trabajos en los que figura mi nombre, y este documento ha seguido el mismo camino.

No era ni es nuestra intención, exponer la sistemática de elaboración del documento en cuestión ni de los futuros, sino sólo publicar el documento final del consenso, y siempre a nivel de recomendaciones, no pretendemos que sea una guía.

Pero en respuesta a las lógicas objeciones de los colegas, y para que en el futuro no haya dudas del método, paso a detallar la sistemática metodológica seguida:

- Se preparó con una metodología de discusión tipo Delphy.

- Se efectuaron 6 reuniones, en las que asistieron más del 90\% de los firmantes en cada una de ellas.

- A cada miembro se le asignó un tema a revisar; se determinó que fueran los artículos de los 5 últimos años sobre el tema, y sólo a nivel excepcional se analizaron algunos que son referencia obligada; en total se revisaron más de 500 artículos.

- En cada reunión se discutían los comentarios de cada miembro, los que se habían recibido previamente por e-mail.

- Todas las sesiones fueron transcritas por una secretaría ajena al grupo: se contrató un MIR de Urología del último año para que hubiera una total fiabilidad en el entendimiento del vocabulario de la discusión; además las sesiones fueron grabadas en audio para mayor exactitud y cotejadas con la transcripción.

- Al iniciarse cada sesión se analizaba críticamente el acta anterior, recibida previamente por e-mail.

- Las reuniones se efectuaron la mayoría en hoteles de Madrid, a puerta cerrada, y sólo dos concretamente, se efectuaron en otros lugares aprovecharon sendos eventos urooncológicos muy interesantes para la inmensa mayoría de los miembros del grupo en relación a su asistencia, la reunión del Grupo de Urología Oncológica (Castelló de la Plana) y Curso de Urología de Sevilla, organizado por el Dr. Marceliano García; las dos reuniones mencionadas se efectuaron el día antes y el día después, respectivamente, en los hoteles sede del evento. 
- La esponsorización fue buscada por el vocal de actividades científicas y yo mismo, y fue a cargo del Lab. Novartis. Quiero explicitar que nunca participó ningún miembro del laboratorio en las discusiones, ni hubo la más minima interferencia en la libertad de criterio de los miembros en ningún momento; que de otra parte ningún miembro de los participantes nunca hubiera aceptado.

- En relación a elección de los expertos, asumo mi responsabilidad en cuanto a derecho de selección, pero creo que estaremos de acuerdo en que son todos los que están, si bien reconozco que no están todos los que son, lógicamente.

Se tituló recomendaciones puesto que ni los niveles de evidencia son siempre tipo 1 , ni existe consenso universalmente aceptado en todos los aspectos, ya que en algunos temas las alternativas son varias, con niveles de evidencia similar, lo que las hace hasta el momento científicamente igual de aceptables, si bien discutibles, por lo que cada uno puede inclinarse por una $u$ otra actitud.

Agradezco que se aprecien estas iniciativas que no son fáciles de realizar ya que suponen una dedicación y esfuerzo muy considerables, siguiendo la doctrina de la Medicina Basada en la Evidencia, y que pretenden enriquecer la urología española.

\section{Fdo.: Dr. A. Gelabert Mas}

Coordinador Nacional.

Grupo de Urología Oncológica de la AEU 